\title{
Visual discrimination learning: Interactions of auditory input pattern, articulation, and instructions '?
}

\author{
STEPHEN L. CARMEAN AND JOSEPH C. MCLACHLAN \\ WESTERN WASHINGTON STATE COLLEGE
}

Visual discrimination learning was affected by the pattem of stimulus names which Ss heard. A positive pattem facilitated, a negative pattern did not effect, and a mixed pattem interfered with discrimination leaming. Interpretation of articulation and instructional effects was complicated by a significant Pattem by Articulation by Instructions interaction which primarily reflected differences between minimally and fully informed, articulating $S$ s with a negative patter and minimally and fully informed, non-articulating $S s$ with a mixed pattern.

Carmean \& Weir (in press) recently found that rate of visual discrimination learning could be enhanced or depressed by having Ss verbalize the stimuli in preselected patterns. It was hypothesized that verbalization affected memory, an assumption supported by other evidence (Mechanic \& D'Andrea, 1965; Murray, 1966) and that memory, in turn, affected discrimination learning.

This study was designed to verify the effects of three of the verbalization patterns used by Carmean and Weir and to determine whether the effects were due to, or independent of, the articulations which their Ss made in generating the auditory input. The term "input" is used to describe this procedure in preference to "feedback" since the auditory stimulation is predetermined and independent of S's response. In addition, an instructional variable was introduced to test the hypothesis that the previously observed failure of Ss with negative input to perform better than controls reflected a failure on the part of these Ss to effectively use all the information which had been stored on previous trials.

\section{Method}

The 204 Ss (introductory psychology students) were assigned in rotation to one of the 12 experimental groups $(\mathrm{N}=12)$ or to the control group $(\mathrm{N}=60)$ with the exception of the last 24 , all of whom were assigned to the control group. Data for five additional Ss were unuseable due to Es' errors or equipment failure.

Ten pairs of animal pictures were rear-projected onto an eye-level screen in modified random order, and $\mathbf{S}$ indicated his choice for each pair by pushing one of the two buttons located just below the screen. Choice of a positive stimulus resulted in the sounding of a singletone door chime. Following each response(non-corrective procedure), blue signal lights would indicate the stimulus $S$ was to name if he were in an articulating group or the name would be delivered via tape recorder if he were not. The tape recorded input intensity measured at the usual position of S's head was approximately $75 \mathrm{~dB}$. With a background level of approximately $50 \mathrm{~dB}$, this was approximately conversational level. Four sec after S's button-pushing response, the signal light would go off or the tape recorder stop, and the filmstrip projector would advance to the next pair of stimuli.

Three patterns of auditory input were used: positive, $S$ heard only the names of correct stimuli; negative, $S$ heard only the names of incorrect stimuli; and mixed, $S$ heard names of both correct and incorrect stimuli with one of the pair present on a given trial preselected at random with the restrictions that each stimulus be verbalized an equal number of times over the 120 trials and that the same member of a given pair not be verbalized on more than two successive occasions.

Instructions were varied orthogonally to the other manipulations. The minimal instructions contained somewhat less information for the experimental Ss than those used by Carmean and Weir while the full instructions contained more. The control condition, minimal instructions and no auditory input, was essentially the same as that used by Carmean and Weir.

The minimal instructions included the information that the purpose of the experiment was to see how quickly $S$ could learn to choose the correct animal each time, that "correct" meant the one which rang the door chime, that the first time through the set $S$ would simply have to guess, and that the same animals were always correct. For full instructions, the pattern of input was explained after the first block of trials, and positive input groups were told to remember the input, negative to remember the input and chose the other stimuli, and mixed to ignore the input.

The learning session was terminated when $S$ reached a criterion of two successive blocks without an error, or when he completed 120 trials. When analyzing the data, Ss reaching the learning criterion were considered to have perfect scores for the remainder of the 120 trials.

Immediately following the conclusion of the task, S was given $3 \mathrm{~min}$ to write down the names of all the stimuli which he had seen. No indication was previously given $S$ that he would be required to do this.

\section{Results}

In scoring the number of correct responses, the results of the first block of trials were discarded, leaving a total of 110 possible correct responses. This was done because learning could not have affected the responses 


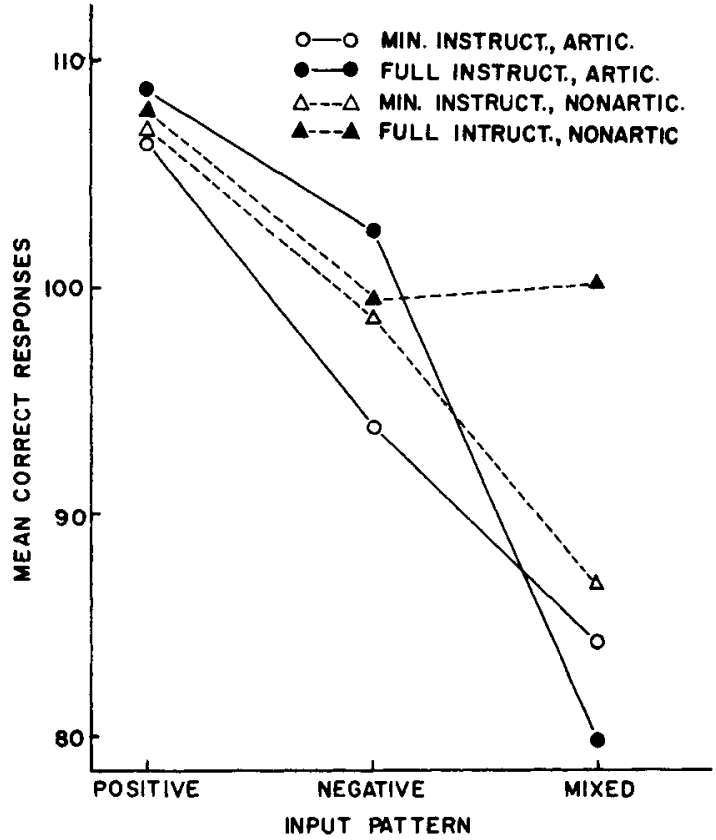

Fig. 1. Mean correct responses of experimental groups.

made in this block, and thus disregarding these data slightly increases the precision of the analysis. The mean number of correct responses for the control group in these 11 blocks was 99.02. Using Dunnett's test for multiple comparisons with a control at the .05 level of significance, the overall mean for positive input groups (107.50) was significantly higher and that for mixed input groups (88.15) significantly lower. The overall mean for negative input groups (98.71) was not significantly different from the control.

The performance of experimental groups as a function of input pattern, articulation, and instructions is summarized in Fig. 1. Analysis of variance revealed that all three variables entered into significant interactions, Pattern by Articulation $(F=3.25, d f=2 / 133, p<.05)$ and Pattern by Articulation by Instructions $(F=3.34$, df $=$ $2 / 133, p<.05)$, and that the main effects of Pattern $(F=30.00, \mathrm{df}=2 / 133, \mathrm{p}<.001)$ and of Articulation $(F=$ 4.08 , df $=1 / 133, p<.05$ ) were also significant. Neither the main effect of Instructions ( $F=2.91$, df $=1 / 133, p<$ .10) nor the interactions of Pattern by Instructions $(F<1, d f=1 / 133)$ or Articulations by Instructions $(F<1$, df $=1 / 133$ ) were significant.

The groups also differed in total recall, recall of correct stimuli, and recall of incorrect stimuli with Stimulus Valence (correct or incorrect) $(F=18.55, \mathrm{df}=$ $1 / 132, \mathrm{p}<.001$ ) and Stimulus Valence by Pattern $(\mathrm{F}=$ 58.85, df $=2 / 132, p<.001$ ) being significant. Neither Articulation, Instructions, nor any of the interactions involving these variables reached the .01 level of significance.

The mean recall scores for the control group were 7.6 correct stimuli and 5.9 incorrect for a total of 13.5.
Using Dunnett's test to compare the retention of each of the input pattern groups with these values, the positive groups remembered more correct stimuli (8.1) and fewer incorrect (5.1), the negative groups fewer correct stimuli (5.8) and more incorrect (7.6), and the mixed groups more incorrect (6.5) and more total stimuli (14.5); all differences significant at the .05 level. Discussion

The similarity of these results to those of Carmean and Weir is taken as convincing evidence of the genuineness of the phenomenon. In both cases, positive input enhanced learning and recall of correct stimuli, negative input did not affect learning but enhanced recall of incorrect stimuli, and mixed input depressed learning and enhanced total recall.

A simple acceptance or rejection of the hypothesis that articulation is essential for the occurrence of the phenomenon is precluded by the significant Pattern by Articulation by Instructions interaction. With a positive pattern, it appears that articulation has no effect above that of auditory input alone. It is possible that a small difference does exist but is masked by a strong ceiling effect since the poorest of these four groups made only 3.6 errors.

With negative input, fully informed Ss made fewer errors - though only if they were articulating. And with mixed input, fully informed Ss made fewer errors-but only if not articulating. These two differences contributed most of the variance in the significant Pattern by Articulation by Instruction interaction, and both were significant at the .05 level using simplet-tests. Neither was significant at the .05 level using Scheffe's test for post-hoc comparisons.

The difference between fully and minimally informed Ss with negative input was expected since it was assumed that full instructions would result in greater utllization of stored negative information. That this occurred only with articulating Ss may mean that articulation does increase storage (although the recall data gave no indication of this), or that non-articulating Ss were for some reason less likely to follow the instructions.

As for the second effect, the full instructions for the mixed pattern included a command to ignore the auditory input. Since this was effective only with non-articulating $\mathrm{Ss}$, It suggests that a person can ignore what he hears, but not what he says.

\section{References}

Carmean, S. L., \& Weir, M. W. Effects of verbalization on discrimination learning and retention. $J$. verbal Learn. verbal Behav., in press.

Mechanic, A., \& $\mathbf{D}$ 'Andrea, J. The role of articulation in response learning. Proc. Amer. psychol. Assoc., 1965, 1, 61-62.

Murray, D. J. Vocalization-at-presentation and immediate recall, with varying recall methods. Quart. J. exp. Psychol., 1966 , $18,9-18$.

\section{Notes}

1. This research was supported by a grant from the Bureau for Faculty Research, Western Washington State College.

2. We thank Pacific Northwest Bell Telephone Co. for generously donating the necessary programming equipment. 